\title{
Some conditions under which derivations are zero on Banach $*$-algebras
}

\author{
Amin Hosseini \\ Department of Mathematics, \\ Kashmar Higher Education Institute, Iran \\ email: A.hosseini@mshdiau.ac.ir, \\ hosseini.amin82@gmail.com
}

\begin{abstract}
Let $\mathcal{A}$ be a Banach *-algebra. By $\mathcal{S}_{\mathcal{A}}$ we denote the set of all self-adjoint elements of $\mathcal{A}$ and by $\mathcal{O}_{\mathcal{A}}$ we denote the set of those elements in $\mathcal{A}$ which can be represented as finite real-linear combinations of mutually orthogonal projections. The main purpose of this paper is to prove the following result:

Suppose that $\overline{\mathcal{O}_{\mathcal{A}}}=\mathcal{S}_{\mathcal{A}}$ and $\left\{\mathrm{d}_{\mathrm{n}}\right\}$ is a sequence of uniformly bounded linear mappings satisfying $d_{n}(p)=\sum_{k=0}^{n} d_{n-k}(p) d_{k}(p)$, where $p$ is an arbitrary projection in $\mathcal{A}$. Then $\mathrm{d}_{\mathrm{n}}(\mathcal{A}) \subseteq \bigcap_{\varphi \in \Phi_{\mathcal{A}}} \operatorname{ker} \varphi$ for each $\mathrm{n} \geq 1$. In particular, if $\mathcal{A}$ is semi-prime and further, $\operatorname{dim}\left(\bigcap_{\varphi \in \Phi_{\mathcal{A}}} \operatorname{ker} \varphi\right) \leq 1$, then $d_{n}=0$ for each $n \geq 1$.
\end{abstract}

\section{Introduction and preliminaries}

In this paper, $\mathcal{A}$ represents a Banach $*$-algebra over the complex field $\mathbb{C}$. If $\mathcal{A}$ is unital, then $\mathbf{1}$ will stand for its unit element. Moreover, $\mathcal{A}$ is called semi-prime if $a \mathcal{A} a=\{0\}$ implies that $a=0$. A non-zero linear functional $\varphi$ is called a character if $\varphi(a b)=\varphi(a) \varphi(b)$ for every $a, b \in \mathcal{A}$. By $\Phi_{\mathcal{A}}$ we denote the set of all characters on $\mathcal{A}$. It is well known that, $\operatorname{ker} \varphi$ the kernel of $\varphi$ is a maximal ideal of $\mathcal{A}$, where $\varphi$ is an arbitrary element of $\Phi_{\mathcal{A}}$. We denote the set of all selfadjoint projections in $\mathcal{A}$ by $\mathcal{P}_{\mathcal{A}}$ (i.e., $\mathcal{P}_{\mathcal{A}}=\left\{p \in \mathcal{A} \mid p^{2}=p, p^{*}=p\right\}$ ), and by $\mathcal{S}_{\mathcal{A}}$ 
we denote the set of all self-adjoint elements of $\mathcal{A}$ (i.e., $\mathcal{S}_{\mathcal{A}}=\left\{a \in \mathcal{A} \mid \mathrm{a}^{*}=\mathrm{a}\right\}$ ). Next, the set of these elements in $\mathcal{A}$ which can be represented as finite reallinear combinations of mutually orthogonal self-adjoint projections, is denoted by $\mathcal{O}_{\mathcal{A}}$. Hence, we have $\mathcal{P}_{\mathcal{A}} \subseteq \mathcal{O}_{\mathcal{A}} \subseteq \mathcal{S}_{\mathcal{A}}$. Note that if $\mathcal{A}$ is a von Neumann algebra, then $\mathcal{O}_{\mathcal{A}}$ is norm dense in $\mathcal{S}_{\mathcal{A}}$. More generally, the same is true for $A W^{*}$-algebras. Recall that a $C^{*}$-algebra is a Banach $*$-algebra in which, for every $a,\left\|a^{*} a\right\|=\|a\|^{2}$. A $W^{*}$-algebra is a weakly closed self-adjoint algebra of operators on a Hilbert space, and an $A W^{*}$-algebra is a $C^{*}$-algebra satisfying: i) In the partially ordered set of projections, any set of orthogonal projections has a least upper bound (LUB),

ii) Any maximal commutative self-adjoint subalgebra is generated by its selfadjoint projections. That is, it is equal to the smallest closed subalgebra containing its self-adjoint projections.

When $\mathcal{A}$ is an $A W^{*}$-algebras it can be proved that each maximal commutative *-subalgebra of $\mathcal{A}$ is monotone complete and $\mathcal{A}$ is unital.

The above-mentioned definitions and results can all be found in [1], [5] and $[10]$ and reader is referred to this sources for more general information on $W^{*}$ algebras and $A W^{*}$-algebras. In this paper, similar to Brešar [1], the author's attention is concentrated on Banach $*$-algebras in which $\mathcal{O}_{\mathcal{A}}$ is norm dense in $\mathcal{S}_{\mathcal{A}}$, i.e. $\overline{\mathcal{O}_{\mathcal{A}}}=\mathcal{S}_{\mathcal{A}}$.

A linear mapping $\mathrm{d}: \mathcal{A} \rightarrow \mathcal{A}$ is called a derivation if it satisfies the Leibnitz's rule $d(a b)=d(a) b+a d(b)$ for all $a, b \in \mathcal{A}$. An additive mapping $d: \mathcal{A} \rightarrow \mathcal{A}$ is called a Jordan derivation if $d\left(a^{2}\right)=d(a) a+a d(a)$ holds for all $a \in \mathcal{A}$. If we define a sequence $\left\{d_{n}\right\}$ of linear mappings on $\mathcal{A}$ by $d_{0}=I$ and $d_{n}=\frac{d^{n}}{n !}$, where I is the identity mapping on $\mathcal{A}$, then the Leibnitz's rule ensures us that $d_{n}$ 's satisfy the condition

$$
d_{n}(a b)=\sum_{k=0}^{n} d_{n-k}(a) d_{k}(b)
$$

for each $a, b \in \mathcal{A}$ and each non-negative integer n. This motivates us to consider the sequences $\left\{d_{n}\right\}$ of linear mappings on an algebra $\mathcal{A}$ satisfying (1). Such a sequence is called a higher derivation. A sequence $\left\{d_{n}\right\}$ of linear mappings on an algebra $\mathcal{A}$ satisfying $d_{n}(p)=\sum_{k=0}^{n} d_{n-k}(p) d_{k}(p)$, where $p$ is an arbitrary element of $\mathcal{P}_{\mathcal{A}}$, is called a pre-higher derivation. A pre-higher derivation $\left\{\mathrm{d}_{\mathrm{n}}\right\}$ is called uniformly bounded if there exists an $M>0$ such that $\left\|d_{n}\right\| \leq M$ for each $n$. In current note, the focus of attention is on uniformly bounded pre-higher derivations. The question under which conditions all derivations are zero on a given $*$-algebra have attracted much attention of authors (for 
instance, see [3], [4], [6], [8], [9], and [12]). In this paper, we also concentrate on this topic. Let us provide a background of our study. In 1955, Singer and Wermer [11] achieved a fundamental result which started investigation into the range of derivations on Banach algebras. The result states that if $\mathcal{A}$ is a commutative Banach algebra and $\mathrm{d}: \mathcal{A} \rightarrow \mathcal{A}$ is a bounded derivation, then $\mathrm{d}(\mathcal{A}) \subseteq \operatorname{rad}(\mathcal{A})$, where $\operatorname{rad}(\mathcal{A})$ denotes the Jacobson radical of $\mathcal{A}$. It is evident that if $\mathcal{A}$ is semi-simple, i.e. $\operatorname{rad}(\mathcal{A})=\{0\}$, then $\mathrm{d}$ is zero. In this paper, we prove that there is not any non-zero bounded derivation from $\mathcal{A}$ into $\mathcal{A}$ without considering the commutativity and semi-simplicity assumptions for $\mathcal{A}$. Indeed, we prove the following result:

Suppose that $\mathcal{A}$ is a semi-prime Banach $*$-algebra so that $\mathcal{O}_{\mathcal{A}}$ is norm dense in $\mathcal{S}_{\mathcal{A}}$, and $\mathrm{d}: \mathcal{A} \rightarrow \mathcal{A}$ is a bounded derivation. If $\operatorname{dim}\left(\bigcap_{\varphi \in \Phi_{\mathcal{A}}} \operatorname{ker} \varphi\right) \leq 1$, then $\mathrm{d}$ is identically zero. In this case, it is possible that $\operatorname{rad}(\mathcal{A}) \neq\{0\}$, and it means that $\mathcal{A}$ is not semi-simple.

Let $\left\{d_{n}\right\}$ be a uniformly bounded pre-higher derivation (i.e., $\left\|d_{n}\right\| \leq M$ for some positive number $M$ ) and $p$ be an arbitrary element of $\mathcal{P}_{\mathcal{A}}$. Then, the function $F$ given by $F(t)=\sum_{n=0}^{\infty} d_{n}(p) t^{n}$ is well defined for $|t|<1$. Indeed,

$$
\begin{aligned}
\left\|\sum_{n=0}^{\infty} d_{n}(p) t^{n}\right\| & \leq \sum_{n=0}^{\infty}\left\|d_{n}(p) t^{n}\right\|=\sum_{n=0}^{\infty}\left\|d_{n}(p)\right\|\left|t^{n}\right| \\
& \leq \sum_{n=0}^{\infty}\left\|d_{n}\right\|\|p\|\left|t^{n}\right| \leq \sum_{n=0}^{\infty} M\|p\|\left|t^{n}\right|<\infty .
\end{aligned}
$$

Moreover, the $\mathrm{m}$-th derivative of $\mathrm{F}$ exists and is given by the formula $F^{(m)}(t):=\sum_{n=m}^{\infty} \frac{n !}{(n-m) !} d_{n}(p) t^{n-m}$. There is a good match between $F(t)$ and the uniformly bounded pre-higher derivation $\left\{d_{n}\right\}$. Using $F(t)$ the following main result is proved:

Let $\mathcal{A}$ be a Banach $*$-algebra such that $\overline{\mathcal{O}_{\mathcal{A}}}=\mathcal{S}_{\mathcal{A}}$. Suppose that $\left\{\mathrm{d}_{\mathrm{n}}\right\}$ is a uniformly bounded pre-higher derivation. Then, $\mathrm{d}_{\mathrm{n}}(\mathcal{A}) \subseteq \bigcap_{\varphi \in \Phi_{\mathcal{A}}} \operatorname{ker} \varphi$ for each $\mathrm{n} \geq 1$. In particular, if $\mathcal{A}$ is semi-prime and further, $\operatorname{dim}\left(\bigcap_{\varphi \in \Phi_{\mathcal{A}}} \operatorname{ker} \varphi\right) \leq$ 1 , then $d_{n}=0$ for each $n \geq 1$.

\section{Results and proofs}

Before proving the main results, we present the following lemma:

Lemma 1 [[1], Lemma 1] Let $\mathcal{A}$ be a normed complex $*$-algebra. If a linear mapping $\delta$ of $\mathcal{A}$ into a normed $\mathcal{A}$-bimodule $\mathcal{M}$ satisfies $\delta(p)=\delta(p) p+p \delta(p)$ 
for all $p \in \mathcal{P}_{\mathcal{A}}$, then $\delta\left(w^{2}\right)=\delta(w) w+w \delta(w)$ holds for all $w \in \mathcal{O}_{\mathcal{A}}$. Moreover, if $\mathcal{O}_{\mathcal{A}}$ is dense in $\mathcal{S}_{\mathcal{A}}$ and $\delta$ is continuous, then $\delta$ is a Jordan derivation.

Note that each member of $\Phi_{\mathcal{A}}$ is continuous (see [2]). Since the case $\Phi_{\mathcal{A}}=\emptyset$ makes everything trivial, so we will assume that $\Phi_{\mathcal{A}}$ is a non-empty set.

Theorem 1 Let $\mathcal{A}$ be a Banach *-algebra such that $\overline{\mathcal{O}_{\mathcal{A}}}=\mathcal{S}_{\mathcal{A}}$. Suppose that $\left\{\mathrm{d}_{\mathrm{n}}\right\}$ be a uniformly bounded pre-higher derivation. Then $\mathrm{d}_{\mathrm{n}}(\mathcal{A}) \subseteq \bigcap_{\varphi \in \Phi_{\mathcal{A}}} \operatorname{ker} \varphi$ for each $\mathrm{n} \geq 1$. In particular, if $\mathcal{A}$ is semi-prime and $\operatorname{dim}\left(\bigcap_{\varphi \in \Phi_{\mathcal{A}}} \operatorname{ker} \varphi\right) \leq 1$, then $\mathrm{d}_{\mathrm{n}}=0$ for each $\mathrm{n} \geq 1$.

Proof. Let $p$ be an arbitrary element of $\mathcal{P}_{\mathcal{A}}$. We know that the function $F(t)=\sum_{n=0}^{\infty} d_{n}(p) t^{n}$ is well-defined for $|t|<1$. Note that

$$
\begin{aligned}
F(t) F(t) & =\left(\sum_{n=0}^{\infty} d_{n}(p) t^{n}\right)\left(\sum_{n=0}^{\infty} d_{n}(p) t^{n}\right)=\sum_{n=0}^{\infty}\left(\sum_{k=0}^{n} d_{n-k}(p) d_{k}(p)\right) t^{n} \\
& =\sum_{n=0}^{\infty} d_{n}(p) t^{n}=F(t) .
\end{aligned}
$$

Hence, $\varphi(F(t))=0$ or $\varphi(F(t))=1$, where $\varphi$ is an arbitrary fixed element of $\Phi_{\mathcal{A}}$. Let $\mathrm{G}(\mathrm{t}):=\varphi(\mathrm{F}(\mathrm{t}))$. We have $\mathrm{G}(\mathrm{t})=\varphi\left(\sum_{n=0}^{\infty} \mathrm{d}_{\mathfrak{n}}(\mathrm{p}) \mathrm{t}^{\mathrm{n}}\right)=\sum_{n=0}^{\infty} \varphi\left(\mathrm{d}_{\mathfrak{n}}(\mathrm{p})\right) \mathrm{t}^{\mathrm{n}}$. It is observed that $G(t)$ is a power series in $\mathbb{C}$. Thus, the $m$-th derivative of $G$ exists and is given by the formula $G^{(m)}(t):=\sum_{n=m}^{\infty} \frac{n !}{(n-m) !} \varphi\left(d_{n}(p)\right) t^{n-m}$. Since the function $G$ is constant, we have $\mathrm{G}^{(\mathrm{m})}(\mathrm{t})=0$ for every $\mathrm{m} \in \mathbb{N} \backslash\{0\}$ and every $|\mathrm{t}|<1$. We have $\varphi\left(\mathrm{d}_{1}(\mathrm{p})\right)+$ $2 \varphi\left(d_{2}(p)\right) t+3 \varphi\left(d_{3}(p)\right) t^{2}+4 \varphi\left(d_{4}(p)\right) t^{3}+\ldots=G^{(1)}(t)=0$. Putting $t=0$ in the former equation, we obtain that $\varphi\left(d_{1}(p)\right)=0$. Using an argument similar to what was described concerning $\varphi\left(d_{1}(p)\right)$, we conclude that $\varphi\left(d_{2}(p)\right)=0$. By continuing this procedure, we prove that $\varphi\left(d_{n}(p)\right)=0$ for all $n \geq 1$. Our next task is to show that $\varphi\left(d_{n}(a)\right)=0$ for every $a \in \mathcal{A}$. Let $x$ be an arbitrary element of $\mathcal{O}_{\mathcal{A}}$. Hence, $x=\sum_{i=1}^{m} r_{i} p_{i}$, where $p_{1}, p_{2}, \ldots, p_{m}$ are mutually orthogonal self-adjoint projections and $r_{1}, r_{2}, \ldots, r_{m}$ are real numbers. We have $\varphi\left(d_{n}(x)\right)=\varphi\left(d_{n}\left(\sum_{i=1}^{m} r_{i} p_{i}\right)\right)=\sum_{i=1}^{m} r_{i} \varphi\left(d_{n}\left(p_{i}\right)\right)=0$. Since $\overline{\mathcal{O}_{\mathcal{A}}}=\mathcal{S}_{\mathcal{A}}$, $\varphi\left(d_{n}(a)\right)=0$ for every $a \in \mathcal{S}_{\mathcal{A}}$. It is well-known that each $a$ in $\mathcal{A}$ can be represented as $a=a_{1}+i a_{2}, a_{1}, a_{2} \in \mathcal{S}_{\mathcal{A}}$; therefore, $\varphi\left(d_{n}(a)\right)=\varphi\left(d_{n}\left(a_{1}+i a_{2}\right)\right)=$ $\varphi\left(d_{\mathfrak{n}}\left(a_{1}\right)\right)+i \varphi\left(d_{n}\left(a_{2}\right)\right)=0$ for all $n \geq 1, a \in \mathcal{A}$ and $\varphi \in \Phi_{\mathcal{A}}$. It means that $\mathrm{d}_{\mathrm{n}}(\mathcal{A}) \subseteq \bigcap_{\varphi \in \Phi_{\mathcal{A}}} \operatorname{ker} \varphi$. Now, suppose that $\operatorname{dim}\left(\bigcap_{\varphi \in \Phi_{\mathcal{A}}} \operatorname{ker} \varphi\right) \leq 1$. It is obvious that if $\operatorname{dim}\left(\bigcap_{\varphi \in \Phi_{\mathcal{A}}} \operatorname{ker} \varphi\right)=0$, then $\mathrm{d}_{\mathrm{n}}(\mathcal{A})=\{0\}$ for all $\mathrm{n} \geq 1$. Assume that $\operatorname{dim}\left(\bigcap_{\varphi \in \Phi_{\mathcal{A}}} \operatorname{ker} \varphi\right)=1$. First we reduce our discussion to the 
case $d_{1}=0$. Since $\operatorname{dim}\left(\bigcap_{\varphi \in \Phi_{\mathcal{A}}} \operatorname{ker} \varphi\right)=1$, there exists a non-zero element $x_{0}$ of $\mathcal{A}$ such that $\bigcap_{\varphi \in \Phi_{\mathcal{A}}} \operatorname{ker} \varphi=\left\{\alpha x_{0} \mid \alpha \in \mathbb{C}\right\}$. Let $\mathrm{a}_{0}$ be an element of $\mathcal{A}$ so that $\mathrm{d}_{1}\left(\mathrm{a}_{0}\right) \neq 0$. We have $\mathrm{d}_{1}\left(\mathrm{a}_{0}\right)=\psi\left(\mathrm{a}_{0}\right) x_{0}$, where $\psi$ is a function from $\mathcal{A}$ into the complex numbers. Having put $\mathrm{b}=\frac{1}{\psi\left(\mathrm{a}_{0}\right)} \mathrm{a}_{0}$, we obtain $\mathrm{d}_{1}(\mathrm{~b})=\mathrm{d}_{1}\left(\frac{1}{\psi\left(\mathrm{a}_{0}\right)} \mathrm{a}_{0}\right)=\frac{1}{\psi\left(\mathrm{a}_{0}\right)} \psi\left(\mathrm{a}_{0}\right) \mathrm{x}_{0}=\mathrm{x}_{0}$ and it implies that $\psi(b)=1$. First we will show $a x_{0}+x_{0} a$ is a scalar multiple of $x_{0}$ for any $a$ in $\mathcal{A}$. Let $a$ be an element of $\mathcal{A}$. Then, $\left.\mathrm{d}_{1}\left(\mathrm{a}^{2}\right)=\psi\left(\mathrm{a}^{2}\right) \mathrm{x}_{0} \quad{ }^{*}\right)$. Lemma 1 is just what we need to tell us that $d_{1}$ is a Jordan derivation, i.e. $d_{1}\left(a^{2}\right)=d_{1}(a) a+a d_{1}(a)$ for all $a \in \mathcal{A}$. Using the fact that $d_{1}$ is a Jordan derivation and the identity $a b+b a=$ $(a+b)^{2}-a^{2}-b^{2}$, we get $d_{1}(a b+b a)=d_{1}(a) b+a d_{1}(b)+d_{1}(b) a+b d_{1}(a)$ for all $a, b \in \mathcal{A}$. Since $d_{1}$ is a Jordan derivation and $\operatorname{dim}\left(\bigcap_{\varphi \in \Phi_{\mathcal{A}}} \operatorname{ker} \varphi\right)=1$, we have $d_{1}\left(a^{2}\right)=d_{1}(a) a+a d_{1}(a)=\psi(a) x_{0} a+a \psi(a) x_{0}=\psi(a)\left(x_{0} a+a x_{0}\right)$ $(* *)$. Comparing $\left(^{*}\right)$ and $\left({ }^{* *}\right)$, we find that $\psi\left(a^{2}\right) x_{0}=\psi(a)\left(a x_{0}+x_{0} a\right)$. If $\psi(a) \neq 0$, then $a x_{0}+x_{0} a=\frac{\psi\left(a^{2}\right)}{\psi(a)} x_{0}$. But if $\psi(a)=0$, then we have

$$
\begin{aligned}
\psi(a b+b a) x_{0} & =d_{1}(a b+b a) \\
& =d_{1}(a) b+a d_{1}(b)+d_{1}(b) a+b d_{1}(a) \\
& =\psi(a) x_{0} b+a \psi(b) x_{0}+\psi(b) x_{0} a+b \psi(a) x_{0} \\
& =a x_{0}+x_{0} a
\end{aligned}
$$

It means that $a x_{0}+x_{0} a$ is a scalar multiple of $x_{0}$ for any a in $\mathcal{A}$. Next, it will be shown that $x_{0}^{2}=0$. Suppose that $\psi\left(x_{0}\right)=0$. We have $\psi\left(b^{2}\right) x_{0}=d_{1}\left(b^{2}\right)=$ $d_{1}(b) b+b d_{1}(b)=\psi(b) x_{0} b+b \psi(b) x_{0}=x_{0} b+b x_{0}$. Applying $d_{1}$ on this equality and then using the fact that $d_{1}\left(x_{0}\right)=\psi\left(x_{0}\right) x_{0}=0$, we obtain that $x_{0}^{2}=0$. Now, suppose $\psi\left(x_{0}\right) \neq 0$. We therefore have

$$
\psi\left(x_{0}^{2}\right) x_{0}=d_{1}\left(x_{0}^{2}\right)=d_{1}\left(x_{0}\right) x_{0}+x_{0} d_{1}\left(x_{0}\right)=2 \psi\left(x_{0}\right) x_{0}^{2} .
$$

If $\psi\left(x_{0}^{2}\right)=0$, then it follows from previous equality that $x_{0}^{2}=0$. Assume that $\psi\left(x_{0}^{2}\right) \neq 0$; so $x_{0}^{2}=\frac{\psi\left(x_{0}^{2}\right)}{2 \psi\left(x_{0}\right)} x_{0}$. Simplifying the notation, we put $\lambda=\frac{\psi\left(x_{0}^{2}\right)}{2 \psi\left(x_{0}\right)}$. Replacing $x_{0}^{2}$ by $\lambda x_{0}$ in $2 \psi\left(x_{0}\right) x_{0}^{2}=d_{1}\left(x_{0}^{2}\right)$, we have $2 \psi\left(x_{0}\right) \lambda x_{0}=\lambda d_{1}\left(x_{0}\right)=$ $\lambda \psi\left(x_{0}\right) x_{0}$. Since $\psi\left(x_{0}\right) \neq 0, \lambda x_{0}=0$ and it implies that either $\lambda=0$ or $x_{0}=0$, which is a contradiction. This contradiction shows that $\psi\left(x_{0}^{2}\right)=0$ and by using (2) it is obtained that $x_{0}^{2}=0$. We know that $x_{0} a+a x_{0}=\mu x_{0}$, where $\mu \in \mathbb{C}$. Multiplying the previous equality by $x_{0}$ and using the fact that $x_{0}^{2}=0$, we see that $x_{0} a x_{0}=0$ for any $a$ in $\mathcal{A}$. Since $\mathcal{A}$ is semi-prime, $x_{0}=0$. From this contradiction we deduce that $d_{1}=0$. Hence, $d_{2}(p)=$ $d_{2}(p) p+p d_{2}(p)+\left(d_{1}(p)\right)^{2}=d_{2}(p) p+p d_{2}(p)$ for every $p \in \mathcal{P}_{\mathcal{A}}$. Reusing 
Lemma 1 , we get $\mathrm{d}_{2}$ is a Jordan derivation. Now, by a procedure similar to what was described concerning $d_{1}$, we obtain that $d_{2}=0$. Consequently, by continuing this procedure, we prove that $d_{n}=0$ for all $n \geq 1$.

An immediate but noteworthy corollary to Theorem 1 is:

Corollary 1 Let $\mathcal{A}$ be a semi-prime Banach *-algebra such that $\overline{\mathcal{O}_{\mathcal{A}}}=\mathcal{S}_{\mathcal{A}}$. If $\operatorname{dim}\left(\bigcap_{\varphi \in \Phi_{\mathcal{A}}} \operatorname{ker} \varphi\right) \leq 1$, then every bounded linear mapping $\mathrm{d}: \mathcal{A} \rightarrow \mathcal{A}$ satisfying $\mathrm{d}(\mathrm{p})=\mathrm{d}(\mathrm{p}) \mathrm{p}+\mathrm{pd}(\mathrm{p})$ for all $\mathrm{p} \in \mathcal{P}_{\mathcal{A}}$, is identically zero.

Proof. First, let us define a sequence $\left\{\mathrm{d}_{\mathrm{n}}\right\}$ of linear mappings on $\mathcal{A}$ by $\mathrm{d}_{0}=$ I and $d_{n}=\frac{d^{n}}{n !}$, where $I$ is the identity mapping on $\mathcal{A}$. A straightforward verification shows that $d_{n}(p)=\sum_{k=0}^{n} d_{n-k}(p) d_{k}(p)$ for all $p \in \mathcal{P}_{\mathcal{A}}$. We have

$$
\left\|d_{n}\right\|=\left\|\frac{d^{n}}{n !}\right\| \leq \frac{1}{n !}\|d\|^{n}<\sum_{n=0}^{\infty} \frac{\|d\|^{n}}{n !}=e^{\|d\|}
$$

for each non-negative integer $n$. It means that $\left\{d_{n}\right\}$ is a uniformly bounded prehigher derivation. It follows from Theorem 1 that $0=d_{1}=d$. Furthermore, Lemma 1 implies that every bounded Jordan derivation from $\mathcal{A}$ into $\mathcal{A}$ is zero.

Remark 1 Let $\left\{\mathrm{d}_{\mathrm{n}}\right\}$ be a higher derivation on an algebra $\mathcal{A}$ with $\mathrm{d}_{0}=\mathrm{I}$, where I is the identity mapping on $\mathcal{A}$. Based on Proposition 2.1 of [7] there is a sequence $\left\{\delta_{n}\right\}$ of derivations on $\mathcal{A}$ such that

$$
(n+1) d_{n+1}=\sum_{k=0}^{n} \delta_{k+1} d_{n-k}
$$

for each non-negative integer $\mathrm{n}$. Therefore, we have

$$
\begin{aligned}
& \mathrm{d}_{0}=I, \\
& \mathrm{~d}_{1}=\delta_{1}, \\
& 2 \mathrm{~d}_{2}=\delta_{1} \mathrm{~d}_{1}+\delta_{2} \mathrm{~d}_{0}=\delta_{1} \delta_{1}+\delta_{2}, \\
& \mathrm{~d}_{2}=\frac{1}{2} \delta_{1}^{2}+\frac{1}{2} \delta_{2}, \\
& 3 \mathrm{~d}_{3}=\delta_{1} \mathrm{~d}_{2}+\delta_{2} \mathrm{~d}_{1}+\delta_{3} \mathrm{~d}_{0}=\delta_{1}\left(\frac{1}{2} \delta_{1}^{2}+\frac{1}{2} \delta_{2}\right)+\delta_{2} \delta_{1}+\delta_{3}, \\
& \mathrm{~d}_{3}=\frac{1}{6} \delta_{1}^{3}+\frac{1}{6} \delta_{1} \delta_{2}+\frac{1}{3} \delta_{2} \delta_{1}+\frac{1}{3} \delta_{3} .
\end{aligned}
$$


Now, assume that $\left\{\mathrm{d}_{\mathrm{n}}\right\}$ is a bounded higher derivation (i.e., $\mathrm{d}_{\mathrm{n}}$ is a bounded linear map for every non-negative integer $\mathrm{n})$. Obviously, $\delta_{1}=\mathrm{d}_{1}$ is bounded. Hence, $\delta_{2}=2 \mathrm{~d}_{2}-\delta_{1}^{2}$ is also bounded. Based on the $\mathrm{d}_{3}$ formula, we have $\delta_{3}=3 \mathrm{~d}_{3}-\frac{1}{2} \delta_{1}^{3}-\frac{1}{2} \delta_{1} \delta_{2}-\delta_{2} \delta_{1}$. Using the boundedness of $\mathrm{d}_{3}, \delta_{1}$ and $\delta_{2}$, we obtain that $\delta_{3}$ is a bounded derivation. In the next step, we will show that every $\delta_{\mathrm{n}}$ is a bounded derivation for every $\mathrm{n} \in \mathbb{N}$. To reach this aim, we use induction on $\mathrm{n}$. According to the above-mentioned discussion, $\delta_{1}, \delta_{2}$ and $\delta_{3}$ are bounded derivations. Now, suppose that $\delta_{\mathrm{k}}$ is a bounded derivation for $\mathrm{k} \leq \mathrm{n}$. We will show that $\delta_{\mathfrak{n}+1}$ is also a bounded derivation. Based on the proof of Theorem 2.3 in [7], we have

$$
\delta_{n+1}=(n+1) d_{n+1}-\sum_{i=2}^{n+1}\left(\sum_{\sum_{j=1}^{i} r_{j}=n+1}(n+1) a_{r_{1}, \ldots, r_{i}} \delta_{r_{1}} \ldots \delta_{r_{i}}\right),
$$

where the inner summation is taken over all positive integers $r_{j}$ with $\sum_{j=1}^{i} r_{j}=$ $\mathrm{n}+1$. From $\sum_{\mathrm{j}=1}^{i} \mathrm{r}_{\mathrm{j}}=\mathrm{r}_{1}+\mathrm{r}_{2}+\ldots+\mathrm{r}_{\mathrm{i}}=\mathrm{n}+1$ along with the condition that $r_{j}$ is a positive integer for every $1 \leq j \leq i$, we find that $1 \leq r_{j} \leq n$ for every $1 \leq j \leq i$. Since we are assuming $d_{n}$ and $\delta_{k}$ are bounded linear mappings for all non-negative integer $\mathrm{n}$ and $\mathrm{k} \leq \mathrm{n}$, it follows from (3) that $\delta_{\mathrm{n}+1}$ is a bounded derivation.

We are now ready for Corollary 2.

Corollary 2 Let $\mathcal{A}$ be a semi-prime Banach $*$-algebra such that $\overline{\mathcal{O}_{\mathcal{A}}}=\mathcal{S}_{\mathcal{A}}$, and $\left\{\mathrm{d}_{\mathrm{n}}\right\}$ be a bounded higher derivation from $\mathcal{A}$ into $\mathcal{A}$. If $\operatorname{dim}\left(\bigcap_{\varphi \in \Phi_{\mathcal{A}}} \operatorname{ker} \varphi\right) \leq 1$, then $\mathrm{d}_{\mathrm{n}}=0$ for all $\mathrm{n} \in \mathbb{N}$.

Proof. Let $\left\{\mathrm{d}_{\mathrm{n}}\right\}$ be the above-mentioned higher derivation. According to Theorem 2.3 of $[7]$ there exists a sequence $\left\{\delta_{n}\right\}$ of derivations on $\mathcal{A}$ such that

$$
d_{n}=\sum_{i=1}^{n}\left(\sum_{\sum_{j=1}^{i} r_{j}=n}\left(\prod_{j=1}^{i} \frac{1}{r_{j}+\ldots+r_{i}}\right) \delta_{r_{1}} \ldots \delta_{r_{i}}\right)
$$

, where the inner summation is taken over all positive integers $r_{j}$ with $\sum_{j=1}^{i} r_{j}=$ $n$. It follows from Remark 1 that $\delta_{\mathfrak{n}}$ is a bounded derivation for every positive integer $n$. At this point, Corollary 1 completes the proof.

Corollary 3 Let $\mathcal{A}$ be a semi-prime Banach $*$-algebra such that $\overline{\mathcal{O}_{\mathcal{A}}}=\mathcal{S}_{\mathcal{A}}$. If $\operatorname{dim}\left(\bigcap_{\varphi \in \Phi_{\mathcal{A}}} \operatorname{ker} \varphi\right) \leq 1$, then $\mathcal{A}$ is commutative. 
Proof. Let $x_{0}$ be a non-zero arbitrary fixed element of $\mathcal{A}$. Define $\mathrm{d}_{\mathrm{x}_{0}}: \mathcal{A} \rightarrow \mathcal{A}$ by $d_{x_{0}}(a)=a x_{0}-x_{0} a$. Obviously, $d_{x_{0}}$ is a bounded derivation. It follows from Corollary 1 that $d_{x_{0}}(a)=0$, i.e. $a x_{0}=x_{0} a$ for all $a \in \mathcal{A}$. Since $x_{0}$ is arbitrary, $\mathcal{A}$ is commutative.

The above results lead us to the following conjecture:

Conjecture 1 Let $\mathcal{A}$ be a semi-prime Banach *-algebra such that $\overline{\mathcal{O}_{\mathcal{A}}}=\mathcal{S}_{\mathcal{A}}$. If $\operatorname{dim}\left(\bigcap_{\varphi \in \Phi_{\mathcal{A}}} \operatorname{ker} \varphi\right)<\infty$, then every bounded derivation from $\mathcal{A}$ into $\mathcal{A}$ is zero.

\section{Acknowledgements}

The author is greatly indebted to the referee for his/her valuable suggestions and careful reading of the paper.

\section{References}

[1] M. Brešar, Characterizations of derivations on some normed algebras with involution, J. Algebra, 152 (1992), 454-462.

[2] H. G. Dales, Banach Algebras and Automatic Continuity, London Math. Soc. Monographs, New Series, 24, Oxford University Press, New York, 2000 .

[3] A. Hosseini, M. Hassani, A. Niknam, On the Range of a Derivation, Iran. J. Sci. Technol. Trans. A Sci. 38 (2014), 111-115.

[4] G. H. Kim, A result concerning derivations in noncommutative Banach algebras, Sci. Math. Jpn., 4 (2001), 193-196.

[5] I. Kaplansky, Projections in Banach Algebras, Ann. of Math., 53 (2) (1951), 235-249.

[6] M. Mathieu, Where to find the image of a derivation, Banach Center Publ., 30 (1994), 237-249.

[7] M. Mirzavaziri, Characterization of higher derivations on algebras, Comm. Algebra, 38 (3) (2010), 981-987.

[8] E. C. Posner, Derivations in prime rings, Proc. Amer. Math. Soc., 8 (1957), 1093-1100. 
[9] N. Shahzad, Jordan and Left Derivations on Locally C*-algebras, Southeast Asian Bull. Math., 31 (2007), 1183-1190.

[10] K. Saitô, J. D. Maitland Wright, On Defining $A W^{*}$-algebras and rickart $C^{*}$-algebras, arXiv: 1501.02434v1 [math. OA] 11 Jan 2015.

[11] I. M. Singer, J. Wermer, Derivations on commutative normed algebras, Math. Ann., 129 (1955), 260-264.

[12] J. Vukman, On derivations in prime rings and Banach algebras, Proc. Amer. Math. Soc., 116 (1992), 877-884.

Received: April 26, 2017 